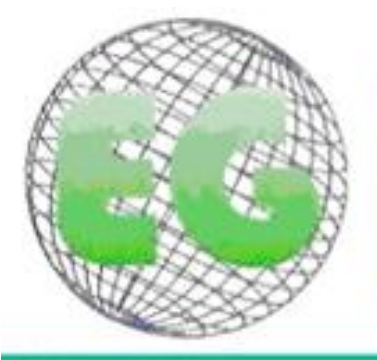

$N^{\circ} 35$

\title{
Presentismo en Enfermería. Implicaciones en seguridad del paciente. Posibilidades de control y reducción
}

Nursing presenteeism. Patient safety implications. Possibilities of control and reduction

\section{${ }^{*}$ Reyes Revuelta, Juan Francisco}

\author{
*Enfermero del servicio COT del Hospital Clínico Universitario Virgen de la Victoria. SAS. Profesor \\ Asociado de la E.U.E. de la Diputación Provincial de Málaga. Universidad de Málaga. E-mail: \\ reyesrevuelta@hotmail.com
}

\begin{abstract}
Palabras clave: Presentismo; Seguridad del Paciente; Salud Laboral; Supervisión de Enfermería. .Keywords: Perfect attendant; Patient Safety; Occupational Health; Nursing supervision.
\end{abstract}

\section{RESUMEN}

Cada vez empezamos a encontrar estudios e investigaciones que orientan hacia la idea de que una peor salud y bienestar de los trabajadores sanitarios puede tener importantes repercusiones para la calidad de los cuidados y la seguridad que los pacientes reciben. El fenómeno emergente del presentismo, referido a aquellos empleados que a pesar de encontrarse enfermos o con alguna lesión que los obligarían a ausentarse, se presentan a trabajar, nos sirve como ejemplo para tratar de explicar esta realidad.

Objetivos: Tratar de responder a las siguientes preguntas: ¿Es enfermería una profesión presentista?, ¿Por qué las enfermeras no cogen la baja por enfermedad?, ¿Qué implicaciones tiene este fenómeno para la Seguridad del Paciente?, ¿Qué posibilidades tienen supervisores de enfermería y gestores de instituciones sanitarias de controlar y reducir el presentismo en sus organizaciones?

Metodología: Se ha realizado una revisión bibliográfica de la evidencia científica disponible en castellano e inglés entre los años 1995 y 2011. Las bases de datos utilizadas han sido Medline, Pubmed, Scopus, Cuiden, CSIC- ISOC y PsycINFO. Esta revisión se completó con búsqueda referencial y búsqueda intuitiva en Google. Encontramos 57 artículos de los que se seleccionaron finalmente 48.

Resultados: La profesión de enfermería, junto con las cuidadoras y la enseñanza a nivel primario son uno de los grupos profesionales con tasas significativamente más altas de presentismo en comparación con otras profesiones. Un gran número de variables, tanto organizacionales como individuales, han sido descritas como predictores del presentismo, es decir, facilitan y aumentan la probabilidad de que una enfermera acuda enferma a trabajar. 
Conclusión. Existe aún un número escaso de investigaciones centradas en el presentismo en el ámbito de instituciones sanitarias. Expertos en salud laboral, gestión sanitaria o en calidad asistencial deberían incluir este nuevo fenómeno en sus agendas de trabajo, diseñando investigaciones para conseguir encontrar una mejor evidencia científica.

\section{ABSTRACT}

We begin to find studies and research oriented towards the idea that poorer health and welfare of health care workers may have important implications for quality of care and safety that patients receive. The emerging phenomenon of presenteeism, referred to those employees who despite being sick or injury that would force them to absent, report to work, serves as an example to try to explain this reality.

Objectives: Try to answer the following questions: Is nursing a presentist profession?, Why nurses do not call in sick? What implications does have this behaviour for Patient Safety? What chance have nursing supervisors and managers of health institutions to control and reduce presenteeism in their organizations?

Methodology: A review of the available scientific evidence literature in Castilian and English between 1995 and 2011 was conducted in order to try to answer the following questions: Database used were Medline, Pubmed, Scopus, Cuiden, CSIC- ISOC y PsycINFO. Search was completed with references and intuitive search in Google. We found 57 items of which 48 were selected.

Results: The nursing profession, with care-and-welfare and education sector at the primary level are one of the professional groups with significantly higher rates of presenteeism compared with other professions. A large number of variables, both organizational and individual have been described as presenteeism predictors, i.e. facilitate and increase the likelihood that a sick nurse go to work.

Conclusions: There is still a small number of research focused on presenteeism in the field of health institutions. Experts in occupational health, health management or quality or care should include this new phenomenon in their agendas, designing research to try to find a better scientific evidence.

\section{INTRODUCCIÓN}

El término presentismo deriva del término en inglés "presenteeism". Se trata de un neologismo para designar un fenómeno que a diferencia del absentismo consiste en que los empleados a pesar de encontrarse enfermos o con alguna lesión que los obligarían a ausentarse y coger la baja por enfermedad, se presentan a trabajar ${ }^{(1,2)}$. Se trata de un fenómeno emergente, de reciente estudio, análisis y descripción cuyo interés ha empezado a tomar un cierto auge al considerar algunos expertos que en términos reales es más perjudicial y mayor el costo originado vía presentismo que vía absentismo ${ }^{(3,4,5,6,7,8)}$. En el año 2004 el prestigioso Harvard Business Review informaba que en Estados Unidos, las pérdidas anuales estimadas por presentismo superaban los 150 billones de US \$ al año, costo que en términos reales sería mayor que el originado por los trabajadores enfermos que permanecen en sus casas ${ }^{(9)}$. Es importante señalar que el término presentismo que se describe no se refiere a un concepto todavía aplicado en algunas legislaciones laborales que premia a aquellos empleados que se ausentan menos en sus trabajos o que no utilizan los permisos que les corresponden (Argentina, Chile y Paraguay). Debe aclararse que tampoco se le debe confundir con la pérdida de tiempo en actividades no productivas en el trabajo (como por ejemplo, ir a tomar café, hacer una pausa para fumar un cigarro, hacer llamadas personales por teléfono) ni con simular estar enfermo para evitar realizar algunas tareas en el trabajo.

La mayoría de los problemas médicos que resultan en presentismo, son por su naturaleza relativamente benignos. Después de todo, las enfermedades más serias 
frecuentemente fuerzan al trabajador a quedarse en casa por períodos extensos de tiempo. Así que las investigaciones en presentismo se han centrado en enfermedades o episodios tales como las alergias estacionales, asma, migrañas y otras clases de cefaleas, dolor de espalda, artritis, infecciones respiratorias, desórdenes gastrointestinales y depresión. Como vemos todas ellas enfermedades con una alta prevalencia, que impactan en la salud, pero que los trabajadores o empleadores no las asumen como algo invalidante o que afecten al desempeño laboral y por tanto a la productividad laboral.

Esta conducta, la de acudir a trabajar estando enfermos, que a algunos les pudiera parecer noble, digna de admiración y ejemplo de un gran profesionalidad puede acarrear consecuencias nefastas, tanto para la salud del propio trabajador como para la calidad de los cuidados ofrecidos y la seguridad del paciente.

El presente artículo tiene como objetivo revisar la literatura científica publicada sobre el presentismo en trabajadores de la salud, específcamente en el colectivo de enfermería y tratar de responder a cuatro cuestiones fundamentales:

¿Es enfermería una profesión presentista?

¿Por qué se da el presentismo entre enfermeras?

¿Qué consecuencias puede acarrear este fenómeno para la seguridad del paciente?

¿Qué posibilidades tienen supervisores de enfermería de gestionar y controlar este fenómeno en beneficio de la seguridad del paciente y de la salud y el bienestar de las enfermeras?

\section{METODOLOGÍA}

Se ha realizado una revisión bibliográfica con el fin de encontrar la evidencia científica disponible sobre presentismo en general y específicamente en el ámbito de la profesión de enfermería así como de estrategias para controlar y gestionar este fenómeno en organizaciones sanitarias.

Estrategia de búsqueda:

Las bases de datos consultadas han sido las siguientes

- Medline

- Pubmed Scopus

- Cuiden

- CSIC-ISOC

- PsycINFO.

Los descriptores de búsqueda utilizados en la bases de datos en inglés han sido:

- "presenteeism",

- "sickness presenteeism",

- "nursing presenteeism",

- "sickness presence", 
- "nursing performance",

- "nursing attendance"

- "sickness attendance".

Los descriptores de búsqueda utilizados en la bases de datos en castellano han sido:

- "presentismo"

- "presentismo \& enfermería"

Criterios de inclusión y exclusión.

- Se seleccionaron artículos con alto nivel de relevancia que abarcan desde el año 1995 al año 2011. La última búsqueda realizada fue el 6 de Noviembre de 2012.

- Se excluyeron los artículos referidos al presentismo en su acepción premio a aquellos empleados que se ausentan menos en sus trabajos o que no utilizan los permisos que les corresponden. También se excluyeron los artículos en su acepción pérdida de tiempo en actividades no productivas en el trabajo.

Tras la búsqueda protocolizada se identificó un total de 57 artículos. Una vez realizada la lectura del título y resumen hemos seleccionados 48 artículos que cumplen con los criterios de inclusión. Tras el análisis del artículo completo hemos seleccionado 32 artículos y hemos añadido 16 artículos de búsqueda referencial y búsqueda intuitiva en Google.

\section{RESULTADOS}

\section{1.-¿Es enfermería una profesión presentista?}

Los estudios realizados durante esta última década han coincidido en señalar a la profesión de enfermería, junto con las cuidadoras y la enseñanza a nivel primario como uno de los grupos profesionales con tasas significativamente más altas de presentismo en comparación con otras profesiones. Un gran estudio, basado en encuestas, realizado entre más de 3300 trabajadores suecos en el año 2000, encontró que las enfermeras eran el grupo profesional con mayor probabilidad de incurrir en presentismo, concretamente 4 veces más que otros grupos profesionales. O.R. 4.26 (IC 95\% 2,05-8,86) ${ }^{(1)}$. En otro estudio realizado en el estado de Tenesee (Estados Unidos) entre 2007 y 2008, la prevalencia de presentismo encontrada en una muestra de 112 enfermeras fue del 50\% ${ }^{(10)}$. Recientemente, en el año 2009, en un estudio llevado a cabo en un Hospital de Nueva Zelanda, el $47 \%$ de las 68 enfermeras encuestadas de forma anónima manifestaron que se presentaron a trabajar con síntomas de enfermedad infecciosa (11). Desafortunadamente no encontramos en la literatura científica ningún estudio publicado sobre presentismo entre enfermeras españolas.

\section{2.-¿Por qué las enfermeras no cogen la baja?}

Se sabe por tanto que las enfermeras trabajan cuando están enfermas, pero se sabe poco de las razones. Un gran número de variables, tanto institucionales, económicas 
como individuales han sido descritas como predictores del presentismo, es decir, facilitan y aumentan la probabilidad de que el trabajador acuda enfermo a trabajar. La profesión de enfermería se caracteriza por poseer varios de estos predictores:

- Altas demandas en el trabajo (presión en el trabajo).

En esta variable se incluyen aquellas características, físicas, cognitivas y sociales de un trabajo que conlleva a prolongar el esfuerzo físico y psicológico de un trabajador. En nuestra profesión nos encontraríamos por ejemplo: bajo control sobre las tareas, altas cargas de trabajo, carencia de tiempo o de recursos, necesidad de estar físicamente presente en el trabajo, necesidad de dar respuesta inmediata a informaciones complejas, numerosas y constantemente diferentes, existencias de situaciones de incertidumbre, trato con pacientes y familiares, existencia de presiones temporales. Demerouti et. al. ${ }^{(12)}$ razonó que aquellos trabajadores que trabajan bajo presión estarían más inclinados al presentismo precisamente para mantener altos niveles de desempeño.

- Alto soporte social por parte de compañeros.

Grynger y Singleton apuntaron la influencia del equipo de trabajo y la presión de los colegas con la decisión de acudir al trabajo enfermo. Altos niveles de cooperación en el desempeño de las tareas se han asociado con altos niveles de presentismo ${ }^{(13)}$. En un estudio cualitativo llevado a cabo en enfermeras australianas se identificó como la principal razón para incurrir en presentismo el fuerte sentido de responsabilidad hacia el equipo. El absentismo era percibido por las enfermeras como negativo y perjudicial hacia sus compañeras. Esta percepción estaba basada en un contexto de plantillas escasas y altas demandas en el trabajo ${ }^{(14)}$.

- Control de la asistencia al trabajo (presión por asistir al trabajo).

Grinyer y Singleton en un estudio cualitativo ilustró cómo aquellas organizaciones que controlaban estrictamente la asistencia al trabajo podía contribuir al presentismo. Especialmente preocupante eran aquellas dinámicas en las que se citaba a un trabajador a una entrevista tras un cierto número de episodios de absentismo que conducían a una acción disciplinaria. Dicho control estimulaba el presentismo ${ }^{(13)}$.

\section{- Inseguridad laboral}

Por un lado cabría pensar que el downsizing (decisión de la organización de reducir el tamaño de su plantilla por razones estratégicas) estimularía el absentismo debido a la percepción de injusticia, rotura del contrato psicológico y estrés que generaría esta situación en el trabajador ${ }^{(15)}$. Por otro lado cabría también pensar que el absentismo se reduciría, como estrategia defensiva, debido al temor a perder el puesto de trabajo, el incremento de la carga de trabajo o que los cambios en la estructura de la organización estimularían la competición entre los trabajadores por hacer más visible su dedicación ${ }^{(16)}$. Implícita o explícitamente la idea es que alguna porción de la reducción en el absentismo conllevaría presentismo.

- Ausencia de pago de los días de baja por enfermedad.

Lovell cita que aquellas empresas con política de que no se pagan los días de baja por enfermedad o sólo se pagan hasta un número limitado de días de baja al año, 
pueden ser un estimulo para el presentismo sobre todo entre las trabajadores con mayores dificultades financieras ${ }^{(17)}$.

\section{- Dificultad o carencia de reemplazo}

Diversas investigaciones han examinado el impacto del presentismo en la dificultad o carencia de reemplazo, entendida como el trabajo perdido debido al absentismo que tiene que recuperar el trabajador después de retornar al trabajo. Estos estudios revelan que los trabajadores están más inclinados a acudir a trabajar a pesar de estar enfermos si ven que el trabajo se les puede acumular ${ }^{(1,18)}$. Esta situación puede ser consecuencia de plantilla escasas, alta especialización o de una carencia de trabajadores polivalentes. McKevitt et al. (19) encontraron que los médicos especialistas en el Reino Unido que trabajaban en Hospitales dieron como respuesta la carencia de reemplazo como la mayor razón para no usar la baja por enfermedad. Después de un período de recortes en funcionarios en Canadá Carvaley et al. encontraron que la carencia de reemplazo fue la razón mayormente citada por presentismo ${ }^{(20) \text {. }}$

\section{- Género femenino.}

El escenario es que las mujeres están más inclinadas al presentismo que los hombres. Aronsson y Gustafsson encontraron que las mujeres estaban de alguna manera más inclinadas que los hombres a informar de presentismo ${ }^{(1,18)}$. Ello puede ser debido por un lado a que las mujeres estaban más representadas en sectores con alto presentismo o bien que la depresión y la migraña, dos de las enfermedades más asociadas con presentismo, son más prevalentes en mujeres que en hombres.

\section{- Cultura presentista}

Trabajos que implican cuidado, ayuda, enseñanza a nivel primario son los más tendentes al presentismo, lo que sugiere una cultura generada en parte a la lealtad hacia usuarios vulnerables como niños y pacientes. La filosofía que tradicionalmente ha rodeado a la identidad enfermera como subordinada y la aparente ausencia de una cultura del cuidado de la propia salud de las enfermeras contribuye en parte también a explicar fenómenos de presentismo ${ }^{(21)}$.

\section{3- ¿Cuál puede ser el impacto del presentismo en la seguridad del paciente?}

En la literatura científica revisada no se ha encontrado ningún estudio de carácter empírico que relacione ambas variables, aunque sí se han encontrado algunos autores que han apuntado la relación que pueda tener el presentismo con la ocurrencia de sucesos adversos en la práctica clínica ${ }^{(22,23,24)}$. El presentismo puede manifestarse en una baja calidad en el trabajo, lo que se traduce en un deterioro de la calidad de los productos y servicios y en que se cometan errores con más frecuencia o de una forma más grave. Además puede manifestarse en una mala administración del tiempo, falta de concentración, pobre trabajo en equipo, en general resulta en un bajo rendimiento por hora trabajada ${ }^{(25)}$.

Los eventos adversos raramente ocurren por un solo error y estos suelen ser la suma de errores latentes o causas básicas del sistema y de errores activos (26). En Seguridad del Paciente el error activo es el término que se utiliza para referirse a errores cometidos por los profesionales en relación directa con los pacientes. Estos 
son, generalmente, fáciles de identificar (pulsar un botón incorrecto, inyectar el medicamento equivocado...) y casi siempre implican a alguien situado en la primera línea asistencial y sus efectos se sienten casi inmediatamente. Entran dentro de esta categoría: los despistes, las distracciones, los lapsus, los errores de valoración y el incumplimiento de normas establecidas. Entre los diversos factores de errores activos se encuentran factores fisiológicos como son: la fatiga, la falta de sueño, el consumo de ciertos medicamentos o el padecimiento de una enfermedad crónica o aguda. De este modo es razonable pensar que una enfermera presentista es más probable que se equivoque y cometa un error activo.

Sí encontramos en la literatura científica un mayor número de artículos que prestan su atención al papel que médicos y otros profesionales sanitarios juegan en la transmisión de enfermedades a los pacientes y al resto de compañeros cuando deciden trabajar a pesar de encontrarse enfermos siendo portadores de enfermedades infectocontagiosas (gripe, gastroenteritis) ${ }^{(24,27,28,29)}$. Como ejemplo del peligroso efecto dominó y de las consecuencias nefastas de lo que un trabajador presentista, portador de una enfermedad infectocontagiosa, puede suponer en un centro sanitario algunos de estos artículos revisados describen la epidemia de Norvovirus en un Hospital de Nueva Zelanda en agosto de 2008, en la que durante un período de 4 semanas más de 383 trabajadores del Hospital y más de 143 pacientes fueron afectados, llegando a perderse más de 2300 turnos de trabajo entre la plantilla del Hospital. El hecho de que hubiera más afectados entre los trabajadores que entre los pacientes hizo pensar que éstos eran portadores y reservorios del virus y que los pacientes pudieron contraer la enfermedad por la interrelación con el personal sanitario presentista.

\section{4- ¿Cómo controlar y reducir el fenómeno del presentismo en su unidad 0 servicio?}

Se hace por tanto necesario que la gestión del riesgo clínico y la prevención de errores activos pase por controlar este nuevo fenómeno ligado al estado de salud de los trabajadores.

De una forma genérica, tal como afirma el investigador chileno Aldo Vera, podíamos aventurar que una respuesta al fenómeno del presentismo sería que las empresas deberían tender a ser organizaciones salutogénicas, generando todos los dispositivos para que las condiciones de trabajo, su organización y su ambiente sean saludables ${ }^{(30)}$. De una forma más concreta algunas de las recomendaciones para supervisores de enfermería o gestores en empresas de salud sobre cómo controlar y reducir el presentismo en beneficio de la Seguridad del Paciente y de la salud y el bienestar de las enfermeras serían las siguientes:

1. Centrar la atención en el presentismo y no únicamente en el absentismo ${ }^{(22,30)}$. Los supervisores que únicamente se centran en el absentismo quizás se encuentren en riesgo de minusvalorar el impacto que pueda tener los trabajadores presentistas en su organización.

2. Familiarizarse con el concepto ${ }^{(22,31)}$. Al tratarse de un fenómeno emergente, cuya compresión, magnitud y repercusión en las organizaciones aún se encuentra en una etapa muy inicial, hace que muchos supervisores y gestores no hayan puesto su atención sobre esta variable con nefastas repercusiones 
sobre la calidad, la seguridad del paciente y la salud y bienestar de las enfermeras.

3. Monitorear los problemas y condiciones de salud física y mental que afectan a las enfermeras y su impacto en el desempeño en el trabajo, midiendo la prevalencia de una serie de patologías comunes incluyendo alergia, migraña, infecciones respiratorias, asma, desórdenes gastrointestinales, artritis, estrés y depresión ${ }^{(31)}$.

4. Hacer estudios entre las enfermeras para cuantificar el presentismo, identificar sus causas y consecuencias, a veces difíciles de detectar. Para ello es posible utilizar una serie cuestionarios autoadministrados con validez y fiabilidad demostrada tales como el Work Limitations Questionnaire (WQL), el Stanford Presenteeism Scale (SPS), el Work Performance Questionnaire (HPQ) y el Work Productivity and Activity Impairment Questionnaire (WPAI) $^{(32,33)}$. A pesar de los sesgos de memoria inherentes a cualquier cuestionario autoadministrado, estas herramientas pueden proporcionar una valiosa información. EI HPQ ha sido desarrollado por la Organización Mundial de la Salud para evaluar los costes indirectos de la salud en el lugar de trabajo y dispone de una versión en castellano llamado Cuestionario sobre Salud y Desempeño (CSD) ${ }^{(7)}$.

5. Realizar campañas de vacunación laboral. Particularmente en el caso de enfermedades infecto-contagiosas y de promoción del lavado de manos y uso de mascarilla ${ }^{(23,34)}$.

6. Potenciar la labor de los Servicios de Medicina Preventiva y de Salud Laboral, que cuentan con profesionales especializados cuyas funciones son la vigilancia de la salud, la prevención primaria y detección precoz de enfermedades y el diseño y desarrollo de programas de promoción de conductas y hábitos saludables entre la población trabajadora (35).

7. Implementar programas de ayuda y asistencia a los empleados con problemas de salud, particularmente depresión, estrés y otros que afecten substancialmente al desempeño laboral ${ }^{(36)}$

8. Evaluar el clima organizacional ${ }^{(36)}$. El fenómeno del presentismo puede expresarse y medirse por señales como:

- Compañeras que piden ayuda más a menudo.

- Compañeras que toman pausas con más frecuencia.

- Compañeras que no son capaces de terminar sus tareas a tiempo.

- Quejas en el equipo por el bajo desempeño de una de sus compañeras.

- Verbalizaciones sobre dificultades e insatisfacción en el trabajo.

- Incremento en las quejas de los pacientes o familiares.

9. El uso de cuestionarios de satisfacción laboral ofrece información adicional para interpretar las causas organizativas que están detrás de fenómenos de presentismo $^{(31)}$. 
10. Establecer políticas claras en relación a la asistencia al trabajo y desalentar el concurrir enfermo a trabajar, particularmente en el caso de enfermedades infecto-contagiosas ${ }^{(23,27,33)}$.

11. Examinar el mensaje explícito o implícito que en ocasiones podemos estar enviado a las enfermeras en aspectos tales como sobrevalorar la presencia del empleado en el trabajo, llegar antes y retirarse después de la hora, controlar estrictamente la asistencia al trabajo, etc. ${ }^{(13,22,23,33)}$.

12. Animar a las enfermeras a que comuniquen sus problemas de salud o enfermedades sin miedo a represalias ${ }^{(13,23,33)}$.

13. Ofrecer a los empleados el pago completo de los días de baja por enfermedad ${ }^{(17)}$

14. Establecer unos ratios adecuados de personal de enfermería en los servicios y asegurar el reemplazo inmediato en caso de compañeros de baja por enfermedad ${ }^{(35)}$

15. Profundizar en el liderazgo mediante un compromiso con la salud de los empleados y la calidad asistencial ${ }^{(35)}$.

16. Hacer de la salud y el bienestar del empleado un objetivo corporativo que sea medible ${ }^{(30)}$.

\section{CONCLUSIÓN}

El bajo desempeño de una enfermera presentista puede estar muchas veces en el origen de los errores activos cometidos en el proceso de cuidado o son la causa de una menor calidad del servicio ofrecido. Existe aún un número escaso de investigaciones centradas en el presentismo en el ámbito de instituciones sanitarias. Expertos en salud laboral, gestión sanitaria o en calidad asistencial deberían incluir este nuevo fenómeno en su agenda de trabajo, diseñando investigaciones donde se relacionen empíricamente ambas variables para conseguir encontrar una mejor evidencia científica.

Es conveniente que supervisores de enfermería y gestores de centros sanitarios superen el modelo centrado únicamente en el absentismo. En primer lugar se recomienda que se familiaricen con el concepto del presentismo y sus nefastas repercusiones y que diseñen estrategias y líneas de actuación encaminadas a su reducción. Con ello no sólo se lograrán menores niveles de presentismo sino también de absentismo, una población trabajadora más sana, más productiva, con mayores niveles de satisfacción laboral y un centro sanitario más seguro para los usuarios.

En el contexto actual de crisis económica se hace necesario indicar que algunas decisiones de carácter político consideradas ajenas a la salud, tienen una influencia directa sobre el presentismo. Hablamos por ejemplo de la supresión del complemento por incapacidad laboral transitoria para los trabajadores de los servicios públicos de salud de algunas comunidades autónomas o la última reforma laboral aprobada en nuestro país, que al facilitar y abaratar el despido contribuye a aumentar la inseguridad de los trabajadores, factor que como hemos visto, se trata de un nítido predictor del presentismo. Otro ejemplo sería el retraso en la edad de jubilación, que 
va a convertir a nuestras enfermeras en un colectivo más envejecido y por tanto con peor estado de salud. Un último ejemplo sería el incremento en los recortes económicos que conducen a una menor carencia de reemplazo de enfermeras y a una mayor presión asistencial, dos variables consideradas predictores del presentismo. La Administración pública por tanto debería estudiar las posibles consecuencias de su acción política sobre fenómenos como el presentismo. Un mayor conocimiento sobre este problema permitiría a los políticos tomar decisiones bien informadas a favor de la salud de los trabajadores, la productividad laboral y la seguridad de los pacientes.

\section{BIBLIOGRAFÍA}

1. Aronsson, G., Gustafsson, K., y Dallner, M.. Sick but yet at work. An empirical study of sickness presenteeism. Journal of Epidemiology and Community Health (2000); 54, 502-509.

2. Dew, K., Keefe, V. y Small, K. 'Choosing' to work when sick: Workplace presenteeism. Social Science \& Medicine (2005); 60, 2273-2282.

3. Burton, W. N., Conti, D. J., Chen, C.-Y., Schultz, A. B. y Edington, D. W. The economic burden of lost productivity due to migraine headache: A specific worksite analysis. Journal of Occupational and Enviromental Medicine (2002); 44, 523-529.

4. Burton, W. N., Pransky, G., Conti, D. J., Chen, C.-Y. y Edington, D. W. The association of medical conditions and presenteeism. Journal of Occupational and Environmental Medicine (2004); 46, S38-S45.

5. Collins, J. J., Baase, C. M., Sharda, C. E., Ozminkowski, R. J., Nicholson, S., Billotti, G. M., et al. The assessment of chronic health conditions on work performance, absence, and total economic impact for employers. Journal of Occupational and Environmental Medicine, (2005); 47, 547-557.

6. Goetzel, R. Z., Long, S. R., Ozminkowski, R. J., Hawkins, K., Wang, S. y Lynch, W. Health, absence, disability, and presenteeism cost estimates of certain physical and mental health conditions affecting U.S. employees. Journal of Occupational and Environmental Medicine (2004); 46, 398-412.

7. Kessler, R. C., Ames, M., Hymel, P. A., Loeppke, R., McKenas, D. K., Richling, D. E., et al. Using theWorld Health Organization Health and Work Performance Questionnaire (HPQ) to evaluate the indirect workplace costs of illness. Journal of Occupational and Environmental Medicine (2004); 46, S23-S37.

8. Koopman, C., Pelletier, K. R., Murray, J. F., Sharda, C. E., Berger, M. L., Turpin, R. S., et al. Stanford Presenteeism Scale: Health status and employee productivity. Journal of Epidemiology and Community Health (2002); 44, 14-20.

9. Hemp, P. Presenteeism: At work-But out of it. Harvard Business Review (2004); 82, 49-58.

10. Warren, C.L. et al.. Cost Burden of the Presenteeism Health Outcome: Diverse Workforce of Nurses and Pharmacists. Journal of Occupational and Environmental Medicine (2011); 53, 90-99.

11. Bracewell, Lisa M et al. Sickness presenteeism in a New Zealand Hospital. Journal of the New Zealand Medical Association (2010); 123, 31-42.

12. Demerouti, E., Le Blanc, P. M., Bakker, A. B., Schaufeli, W. B. y Hox, J. Present but sick: A three-wave study on job demands, presenteeism and burnout. Career Development International (2009); 14, 50-68.

13. Grinyer, A. Singleton, V.. Sickness absence as risk-taking behaviour: A study of organizational and cultural factors in the public sector. Health, Risk \& Society, (2000); 2, 7-21. 
14. Crout, L. A., Chang, E., Cioffi, J. Why do registered nurses work when ill?. Journal of Occupational Nurse Association (2005); 35, 23-35.

15. Kammeyer-Mueller, J., Liao, H., y Arvey, R. D. Downsizing and organizational performance: A review of the literature from a stakeholder perspective. Research in Personnel and Human Resources Management (2001); 20, 269-329.

16. Simpson, R.. Presenteeism, power and organizational change: Long hours as a career barrier and the impact on the working lives of women managers. British Journal of Management (1998); 9, 37-50.

- Lovell, V. No time to be sick: Why everyone suffers when workers don't have paid sick leave. Washington, DC: Institute for Women's Policy Research (2004).

18. Aronsson, G., y Gustafsson, K. Sickness presenteeism: Prevalence, attendancepressure factors, and an outline of a model for research. Journal of Occupational and Environmental Medicine (2005); 47, 958-966.

19. McKevitt, C., Morgan, M., Dundas, R. y Holland, W. W. Sickness absence and "working through" illness: A comparison of two professional groups. Journal of Public Health Medicine (1997), 19, 295-300.

20. Caverley, N., Cunningham, J. B. y MacGregor, J. N. Sickness presenteeism, sickness absenteeism, and health following restructuring in a public service organization. Journal of Management Studies (2007); 44, 304-319.

21. Boughn S, Lentini A. Why do women choose nursing? Journal of Nursing Education (1999); 38, 156-161.

22. Flores-Sandí G. "Presentismo": Potencialidad en accidentes de salud. Acta Médica Costarricense (2006); 48, 30-34.

23. Rosvold, E. O. y Bjertness, E. Physicians who do not take sick leave: hazardous heroes?. Sandinavian Journal fo Public Health (2001); 29, 71-75.

24. Yamasshita, M. y Arakida M. Concept, analysis of presenteeism and its posible applications in Japanese occupational health. Sangyo Eiseigaku Zasshi (2006); 48, 201-213.

25. Marlowe, J. (2007) Presenteeism: limited awareness.Big bottom-line impact. Disponible en Internet. (accesado el 15 de agosto de 2012).

26. Reason J. (1990) Human Error. New York. Cambridge University Press.

27. Gudgeon P., Wells D.A., Baerlocher M.O. et al. Do you come to work with a respiratory tract infection?. Journal of Occupational and Enviromental Medicine (2009); 66, 424.

28. Waldron H.A. Sickness in the Medical Profession. Archives of Occupational Hygiene (1996); 40, 391-396.

29. Widera E., Chang A., Chen H.L. Presenteeism a Public Health Hazard. Journal of General Internal Medicine (2010); 25(11) 1244-1247.

30. Vera A. Presentismo ocasionaría millonarias pérdidas en Chile. Ciencia y Trabajo (2007); 24, A44-A47.

31. Rojas R. Enfoque del presentismo en empresas de salud. Ciencia y Trabajo (2007); 24, 64-68

32. Loflland J.H., Pizzi L., Frick K.D. A Review of Health-related Workplace productivity Loss Instruments. Pharmaeconomics (2004); 22 (3): 165-184

33. Brooks A., Hagen E., et al. Presenteeism: Critical Issues. Journal of Occupational and Enviromental Medicine (2010). 52, 1055-1067.

34. Landry M., Miller C. Preseenteism: Are we Hurting Patiens We are trying To Help?. Journal of General Internal Medicine (2010); 25, 1142-1143.

35. Registered Nursed Association of Ontario. Workplace Health, Safety and Wellbeing of the Nurse (2008). Disponible en internet en http://rnao.ca/sites/rnaoca/files/Workplace Health Safety and Well being of the Nurse.pdf (accesado el 13 de octubre de 2012). 
35. Pilette P.C., Presenteesim in Nursing: A clear and Present Danger to Productivity. Journal of American Nursing Association (2005); 35, 300-3003. 